\title{
RX J1856.5-3754: A Strange Star with a Solid Quark Surface?
}

\author{
X. L. Zhang, ${ }^{1}$ R. X. Xu ${ }^{2}$ \& S. N. Zhang ${ }^{1,3}$ \\ ${ }^{1}$ University of Alabama, Huntsville, AL 35899, USA \\ ${ }^{2}$ School of Physics, Peking University, Beijing 100871, China \\ ${ }^{3}$ Physics Department, Tsinghua University, Beijing 100084, China
}

\begin{abstract}
Within the realms of possibility of solid quark matter, we fitted the $500 \mathrm{ks}$ Chandra LETG/HRC data for RX J1856.5-3754 with a phenomenological spectral model, and found that the electric conductivity of quark matter on the stellar surface is about $>1.2 \times 10^{18} \mathrm{~s}^{-1}$.
\end{abstract}

\section{Introduction}

Neutron stars provide a unique opportunity for researchers who are interested in obtaining experimental information on matter at extremely high density, especially on density-dominated quark matter if a special kind of neutron stars, the so called strange stars, exist. However, the identification of strange stars still faces many challenges, and has become a hot topic in astrophysics.

The featureless spectra of isolated "neutron" stars may be evidence for bare strange stars (Xu 2002), but a definitive conclusion on the nature of these objects cannot be reached until theoretically calculated spectra of the bare quark surface are known. However, due to the strong nonlinearity of quantum chromodynamics, it is almost impossible to present a definitive and accurate calculation of the density-dominated quark-gluon plasma from first principles. Nevertheless, it has been suggested that cold quark matter with an extremely high baryon density could be in a solid state (Xu 2003). We here try to fit the thermal X-ray spectrum of the isolated neutron star RX J1856.5-3754 in this regime.

\section{Fitting the Data and Results}

In solid quark matter, the interactions between electrons, or between electrons and photons, could be responsible for the thermal photon radiation, which could be analogous to the radiation of metals to some extent. We fit the thermal spectrum obtained from Chandra LETG/HRC observations of RX J1856.5-3754 (total exposure time about $500 \mathrm{ks}$ ) with the metal emissivity (Born \& Wolf 1980) $\psi(\nu, T)=\alpha(\nu) B(\nu, T)$, where $\alpha(\nu)=1-(2 \sigma / \nu+1-2 \sqrt{\sigma / \nu}) /(2 \sigma / \nu+1+2 \sqrt{\sigma / \nu})$ and $B(\nu, T)$ is the blackbody emissivity.

Chandra LETG/HRC has performed five observations of RX J1856.5-3754, for a total exposure time $\sim 500 \mathrm{ks}$. Starting from the event2 files and following the CIAO threads, we extracted spectra, generated the corresponding effective area files for each observation, and combined them for fitting. The positive and negative order spectra were combined, and a joint fit was performed. To avoid the artificial features across the gaps between CCDs, we ignored 0.18-0.21 keV 
in the positive order and $0.21-0.26 \mathrm{keV}$ in the negative order. The spectra were grouped so that each channel contained at least 300 counts. We fit the spectrum with both a pure blackbody model and a metal thermal model, both with interstellar absorption. The fitting result is shown is Table 1.

Table 1. Fitting result: (a) absorbed blackbody, (b) absorbed metal thermal spectrum. In (b), the lower limits of $\sigma$ and $R_{\mathrm{bb}}^{\infty}$ are 1-, 2- and 3- $\sigma$ confidence levels, respectively.

\begin{tabular}{cccccc}
\hline Model & $\begin{array}{c}n_{H} \\
\left(10^{20} \mathrm{~cm}^{-1}\right)\end{array}$ & $\begin{array}{c}k T_{\mathrm{bb}}^{\infty} \\
(\mathrm{eV})\end{array}$ & $\begin{array}{c}R_{\mathrm{bb}}^{\infty} \\
(\mathrm{km}[d / 120 \mathrm{pc}])\end{array}$ & $\begin{array}{c}\sigma \\
10^{18} \mathrm{~s}^{-1}\end{array}$ & $\chi^{2} / \mathrm{dof}$ \\
\hline (a) & $0.86 \pm 0.03$ & $64.1 \pm 0.5$ & $4.1 \pm 0.1$ & - & $830 / 970$ \\
(b) & $0.72 \pm 0.03$ & $59.5 \pm 0.5$ & $>(7.4,5.9,4.9)$ & $>(1.24,0.38,0.14)$ & $809 / 969$ \\
\hline
\end{tabular}

The metal model provides a slightly better fit to the data. Since $\sigma$ and $R_{\mathrm{bb}}^{\infty}$ are coupled together, we can only determine lower limits for the value of $\sigma$ or $R_{\mathrm{bb}}^{\infty}$ from the fitting. The parameters for the absorbed blackbody model are a little different from those obtained from the same observations by Burwitz et al. (2003). A possible reason is that we used different binning of the spectra. However, the lower limits of neither $R_{\mathrm{bb}}^{\infty}$ nor $\sigma$ are sensitive to the binning.

\section{Discussion}

Within the realms of solid quark surfaces, we have fitted the $500 \mathrm{ks}$ Chandra LETG/HRC data for the brightest isolated neutron star, RX J1856.5-3754, with a phenomenological spectral model. However, the UV-optical excess (e.g., Burwitz et al. 2003) has not been included in the fitting. Actually the origin of this excess is still academically controversial. We suggest that the emission is not from the stellar surface. During a propeller phase with low accretion from the interstellar medium, a quasistatic atmosphere (envelope) may form around the magnetopshere of RX J1856.5-3754. The dissipation of stellar rotation energy may heat the envelope, which could be responsible for the UV-optical emission. Detailed investigation of this issue is necessary if one wants to understand the environmental status of isolated neutron stars.

If the electrons near the Fermi surface are responsible for the conduction, the fitted $\sigma$ implies a relaxation time $\tau \gtrsim 8 \times 10^{-21} \mathrm{~s}$, while the $e-e$ collision timescale is $\tau \sim 2.3 \times 10^{-16} \mathrm{~s}$. The conductivity fitted could be reasonable.

Strong magnetic fields and/or fast rotation may help a neutron star to reproduce a featureless spectrum. However, the thermal radiation mechanism is very different from ours, which results in a possible future test.

Acknowledgments. This work is supported by NSFC (10273001) and the Special Funds for Major State Basic Research Projects of China (G2000077602).

\section{References}

Born, M., \& Wolf, E. 1980, Principles of Optics, (New York: Pergamon Press) Burwitz, V., et al. 2003, A\&A, 399, 1109

Xu, R. X. 2002, ApJ, 570, L65

Xu, R. X., 2003, ApJ, 596, L59 